\title{
Horizontal Restacking Control of Covalent Organic Framework Nanosheets for Ultrathin Gas Separation Membranes
}

\author{
Yingjie Zhao ${ }^{1}$, Yunpan Ying ${ }^{2}$, Peng $\mathrm{Liu}^{1}$, Kunpeng $\mathrm{Wei}^{1}$, Dan Zhao ${ }^{2}$, and Dahuan $\mathrm{Liu}^{1}$ \\ ${ }^{1}$ Beijing University of Chemical Technology \\ ${ }^{2}$ National University of Singapore
}

November 9, 2020

\begin{abstract}
Covalent organic frameworks (COFs) are a family of promising membrane materials because of their intrinsic pores with uniform size, tunable functionalities, and high stability. Especially, two-dimensional (2D) COFs can be exfoliated into singleor few-layered nanosheets and restacked into molecular sieving membranes through controlling restacking modes and thickness. However, the traditional methods mainly rely on increasing the thickness (vertical restacking control) to achieve high separation selectivity but with limited permeance. Herein, the fabrication of ultrathin COF membranes through a horizontal restacking control with the aid of introducing thermal perturbation is reported, i.e., a heating vacuum filtration method to optimize the relative displacement of nanosheets in horizontal direction without sacrificing thickness control. The obtained membrane exhibits high $\mathrm{H} 2$ permeance (655.6 GPU) and H2/CO2 selectivity (43.2) for CO2 pre-combustion capture. Furthermore, this method also displayed its universality to control the restacking microstructures of other COF nanosheets and the resulted properties of membranes.
\end{abstract}

Horizontal Restacking Control of Covalent Organic Framework Nanosheets for Ultrathin Gas Separation Membranes

Yingjie Zhao, ${ }^{1, \#}$ Yunpan Ying, ${ }^{2, \#}$ Peng Liu,,${ }^{1, \#}$ Kunpeng Wei, ${ }^{1}$ Dan Zhao, ${ }^{2}$ and Dahuan $\mathrm{Liu}^{1 *}$

1 State Key Laboratory of Organic-Inorganic Composites, Beijing Advanced Innovation Center for Soft Matter Science and Engineering, Beijing University of Chemical Technology, Beijing 100029, PR China

${ }^{2}$ Department of Chemical and Biomolecular Engineering, National University of Singapore, 4 Engineering Drive 4, Singapore 117585, Singapore

\# These authors contributed equally.

\section{Corresponding author}

*E-mail: liudh@mail.buct.edu.cn

Abstract: Covalent organic frameworks (COFs) are a family of promising membrane materials because of their intrinsic pores with uniform size, tunable functionalities, and high stability. Especially, two-dimensional (2D) COFs can be exfoliated into single- or few-layered nanosheets and restacked into molecular sieving membranes through controlling restacking modes and thickness. However, the traditional methods mainly rely on increasing the thickness (vertical restacking control) to achieve high separation selectivity but with limited permeance. Herein, the fabrication of ultrathin COF membranes through a horizontal restacking control with the aid of introducing thermal perturbation is reported, i.e., a heating vacuum filtration method to optimize the relative displacement of nanosheets in horizontal direction without sacrificing thickness control. The obtained membrane exhibits high $\mathrm{H}_{2}$ permeance (655.6 GPU) and $\mathrm{H}_{2} / \mathrm{CO}_{2}$ selectivity (43.2) 
for $\mathrm{CO}_{2}$ pre-combustion capture. Furthermore, this method also displayed its universality to control the restacking microstructures of other $\mathrm{COF}$ nanosheets and the resulted properties of membranes.

Keywords: covalent organic frameworks, gas separation, graphene oxide, restacking control, twodimensional membranes

Introduction

Separation technology is one of the critical processes in energy, chemical industry, environment and other related fields. ${ }^{1}$ With the energy crisis and the expansion of industrial production, there is an urgent need to develop efficient separation processes. ${ }^{2}$ Membrane separation has been widely used in gas separation/purification, ${ }^{3}$ water treatment, ${ }^{4}$ seawater desalination, ${ }^{5}$ and organic solvent nanofiltration ${ }^{6}$ because of its advantages including low-energy consumption and easy preparation. ${ }^{7}$ The main challenge for the membrane development is the trade-off between selectivity and permeability, which can be relieved by reducing membrane thickness. ${ }^{8}$ Two-dimensional (2D) nanosheet-based ultrathin membranes have shown excellent separation performances because of their ultrathin thickness (typically less than $500 \mathrm{~nm}$ ) and molecular sieving properties. ${ }^{9}$ The building units of membranes can directly affect the chemical structure and separation performance of membranes. A great number of $2 \mathrm{D}$ nanomaterials have been used to prepare ultrathin membranes, including graphene oxide $(\mathrm{GO}),{ }^{10}$ inorganic materials (zeolites, ${ }^{11}$ transition metal dichalcogenides, ${ }^{12}$ transition metal carbides or carbonitrides, ${ }^{13}$ layered double hydroxides (LDHs) ${ }^{14}$ ), microporous polymers, ${ }^{15}$ metal-organic frameworks (MOFs), ${ }^{16-19}$ and covalent organic frameworks (COFs). ${ }^{20-23}$ Among them, 2D COFs, a kind of porous crystalline organic framework materials connected by in-plane strong covalent bonds and out-plane weak molecular interactions (van der Waals interaction and $\pi-\pi$ stacking interaction), ${ }^{24}$ have attracted gradual attentions due to their high thermal and chemical stability, well defined and highly tunable pore size and layered structures, ${ }^{25,26}$ which can be successfully exfoliated into single- or few-layered COF nanosheets $(\mathrm{CONs}){ }^{27}$ However, there are very rare reports of $2 \mathrm{D}$ COF based membranes for gas separation up to now. The main reason is that the pore size of most COFs $(>0.6 \mathrm{~nm})$ is much larger than the kinetic diameters of common small gas molecules $(<0.5 \mathrm{~nm})$, besides the difficulties in preparing continuous and defect-free COF membranes. ${ }^{28}$

Using our self-developed computational methods, we have revealed that ultrathin 2D COF membranes can form an energetic microenvironment around the narrow interlayer passages through tuning restacking mode (along vertical or horizontal direction to the nanosheets and the surface of support) to introduce interacting surfaces to generate van der Waals potential sites near these passages. ${ }^{29}$ Such few-layered 2D COF membranes can exhibit nonselective to highly selective performance, even to the precisely molecular sieving separation. This strategy provides a new theoretical guidance for experimentally preparing ultrathin membranes with excellent gas separation performance. Accordingly, we have proposed a GO-assisted Layerby-Layer (LbL) restacking method to prepare ultrathin COF/GO membranes. ${ }^{30}$ Continuous and defectfree ultrathin COF/GO membranes with tunable thickness (100-290 nm) have been successfully fabricated with both high gas permeance and high selectivity, achieving tunable separation performance by restacking different amounts of nanosheets longitudinally (vertical direction to the support). Unfortunately, regulating separation performance of $2 \mathrm{D} \mathrm{COF}$ ultrathin membranes in horizontal direction under a constant thickness has not been realized so far, which, if can be realized, may provide more opportunities for tuning the selectivity and permeability of membranes in a wide range to meet the practical requirements. Herein, we use a facile LbL assembly method under heating disturbing process to fabricate 2D COF ultrathin membranes with different horizontal restacking modes. The key concept of this method is the utilization of heating in vacuum filtration process to introduce the perturbation to change the restacking of nanosheets (Scheme 1 ), achieving the target of improving membrane separation performance under a constant thickness as proposed in our theoretical design. In principle, this method also has great potential to optimize other 2D-material-based ultrathin hybrid membranes.

\section{Methods}

Preparation of COF Nanosheets (CONs). CTF-BTD was prepared according to the procedure given in 
our previous work. ${ }^{30}$ The monomer 2,1,3-benzothiadiazole-4,7-benzenedicarbonitrile (BTD) was synthesized using the reported method. ${ }^{31}$ Zinc chloride and BTD were ionothermally reacted at $673 \mathrm{~K}$ after being vacuum-sealed in an ampoule. The black bulk CTF-BTD particles was obtained, after being subsequently washed three times using dilute hydrochloric acid and dichloromethane respectively, and dried at $423 \mathrm{~K}$ in vacuum oven overnight. To prepare nanosheets, $200 \mathrm{mg}$ of CTF-BTD bulk samples and $20 \mathrm{~mL}$ methanol as solvent were added to a ball-milling jar with $3 \mathrm{~mm}$ diameter zirconia balls. The jar was fixed in a ball mill (QM-3SP04, Nanjing NaDa instrument equipment Co., Ltd), and the mixture underwent wet ball milling at a mild speed of $60 \mathrm{rpm}$ for $6 \mathrm{~h}$. The powders can be obtained by drying at $423 \mathrm{~K}$ under vacuum overnight after methanol washing and filtration. CTF-BTD powders were dispersed in methanol solution $(2 \mathrm{mg} / \mathrm{mL}$ ) with stirring for $1 \mathrm{~h}$, then the mixture was sonicated (Power: $250 \mathrm{~W}$; Frequency: 40 $\mathrm{KHz}$ ) (KQ-250DE, KunShan ultrasonic instrument Co., Ltd, China) at room temperature for $1 \mathrm{~h}$ to obtain exfoliated CTF-BTD nanosheets. The dispersion liquid is allowed centrifugation at $8000 \mathrm{rpm}$ to obtain abundant CTF-BTD nanosheets from the supernatant. Finally, the nanosheets were dispersed in methanol for the following membrane preparation and characterization. For GO nanosheets, the $0.01 \mathrm{mg} / \mathrm{mL}$ of GO nanosheets dispersion solution was obtained by ultra-sonicating $1 \mathrm{~mL}$ of GO dispersion $(2 \mathrm{mg} / \mathrm{mL}$, Shanxi Institute of Coal Chemistry, Chinese Academy of Sciences) and $199 \mathrm{~mL}$ of deionized water. The solution was sonicated for $0.5 \mathrm{~h}(250 \mathrm{~W}, 40 \mathrm{KHz})$ and the obtained GO aqueous dispersion was used for membranes preparation.

Ultrathin COF/GO Membranes Fabrication. In a facile membrane preparation process, various amounts GO aqueous dispersion were mixed with $0.2 \mathrm{mg}$ CTF-BTD nanosheets dispersion liquid $(0.24 \mathrm{mg} / \mathrm{mL})$. The mixture solution was then diluted to $250 \mathrm{~mL}$ of filtrate solution $(50 / 50 \mathrm{vol} \%$ of methanol/deionized water) and further sonicated for $20 \mathrm{~min}$. The CTF-BTD/GO membrane was obtained by Layer-by-Layer restacking of the mixed nanosheets dispersion liquid onto an anodic aluminum oxide (AAO) support (pore size: $200 \mathrm{~nm}$ ) using a vacuum filtration system at 1 bar for $2 \mathrm{~h}$. In the vacuum filtration process, the membrane was prepared at different temperatures $(293,333,373,413$ and $453 \mathrm{~K})$ by a simple home-made heating apparatus. After the filtrate being fully filtrated out, the CTF-BTD/GO membrane was dried at $313 \mathrm{~K}$ for $24 \mathrm{~h}$ in a dynamic vacuum oven. The pure GO membranes were prepared under the same conditions but without the adding of CTF-BTD nanosheets. The prepared membranes were denoted as CTF-BTD/GO-X-Y-Z, where X represents that the membrane composed X mg of CTF-BTD nanosheets, Y represents that the membrane composed $\mathrm{Y} \mathrm{mg}$ of GO nanosheets, and $\mathrm{Z}$ represents the membrane preparation temperature $(\mathrm{K})$. CTF-BTD-X-Z represents the membrane prepared by X mg of CTF-BTD nanosheets at Z K. GO-Y-Z represents the membrane is prepared by Y mg of GO nanosheets at Z K.

Characterization Methods. Powder X-ray diffraction (PXRD) were conducted on an X-ray diffractometer (Bruker D8 ADVANCE) in reflection mode using a voltage of $40 \mathrm{kV}$ and a current setting of $40 \mathrm{~mA}$ with a $\mathrm{Cu} \mathrm{K} \alpha(\lambda=1.5406)$ radiation. The $2 \vartheta$ ranging from $5^{\circ}$ to $90^{\circ}$ was scanned with a step size of $0.02^{\circ}$. The $\mathrm{N}_{2}$ adsorption-desorption isotherm at $77 \mathrm{~K}$ and the BET surface area and pore size distributions by DFT method were measured on an automated gas sorption analyzer (3H-2000PM, BeiShiDe Instruments). Before measurement, the sample was degassed at $423 \mathrm{~K}$ overnight. Fourier transform infrared spectroscopy (FTIR) characterization were performed on an FTIR spectrophotometer (Nicolet 6700, Thermo Fisher), and spectra were recorded in the wave number range of 500-3000 $\mathrm{cm}^{-1}$. Raman Spectroscopy were performed on a Renishaw Micro-Raman Spectroscopy System (Renishaw in Via), and excitation wavelength is $785 \mathrm{~nm}$, Raman shift range is $120^{\sim} 3200 \mathrm{~cm}^{-1}$. The morphologies and thicknesses of nanosheets were characterized using an atomic force microscope (AFM, DMFASTSCAN2-SYS). Before AFM inspection, two drops of the diluted nanosheets suspension (1:100 times dilution of original solution) were transferred onto Si wafer and dried at $353 \mathrm{~K}$ for $10 \mathrm{~h}$. The morphologies of bulk samples or nanosheets and the crystalline property was observed and confirmed by transmission electron microscopy (TEM, JEM-2100F, JEOL). Before electron microscopy inspection, one drops of the diluted samples in $\mathrm{CH}_{3} \mathrm{OH}$ solvent were transferred onto cooper grid and dried at room temperature. The cross-sectional lamella for scanning transmission electron microscopy (STEM) were prepared by Focused Ion Beam (FIB, FEI Helios G4 CX) and the cross-sectional morphology were observed on an aberration corrected transmission electron microscope (FEI Tecnai F20). Scanning electron 
microscopy (SEM) were performed by HITACHI S-4800. All samples were mounted on tape and coated with a 1.5-2 $\mathrm{nm}$ Au layer to increase the conductivity, and the measurements were performed under a $10-20 \mathrm{kV}$ acceleration voltage. The surface elemental analysis of the membranes and powder were analyzed by X-ray photoelectron spectroscopy (XPS, KRATOS AXIS SUPRA, Shimadzu). The solid fluorescence analysis of membranes was characterized by fluorescence spectrometer (F-7000, Hitachi) and excitation wavelength was measured by UV-visible spectrophotometer (UV-3600, Shimadzu). The lateral sizes of these nanosheets were also analyzed by dynamic light scattering (DLS, ZS90)

Gas Permeation Experiments. For single gas permeation, the membranes with an effective membrane surface area of $0.785 \mathrm{~cm}^{2}$ were sealed in a home-made soap-bubble flow apparatus with silicone O-rings, ${ }^{32}$ the schematic diagram of the experimental setup for single-gas permeation tests is shown in Fig. S1 (Supporting Information). The feeding gas was fed to the top side of the membranes and the permeate gas flowed through the soap bubble tube. The time was recorded for a certain volume of gas flow under 1.0 bar of pressure difference at room temperature. The permeate side pressure in the system was maintained at the atmosphere pressure. Each of the permeation measurement result was determined once the steady performance had been reached and tested at least three times under each operating condition. The ideal selectivity of gas pair was calculated as the ratio of their permeances. The permeance $(P)$ and ideal selectivity $(I S)$ are defined by the following equations (Eqs. 1-2),

$P_{i}=\frac{N_{i}}{3.348 \times 10^{-10} \cdot \Delta \Pi \cdot A}($ Eq.1)

$I S=\frac{P_{i}}{P_{j}}($ Eq. 2$)$

where $P_{i}$ is the permeance of component gas $i$ (GPU), $N_{i}$ is gas permeation rate ( $\left.\mathrm{mol} \mathrm{s}^{-1}\right), \Delta \Pi$ is transmembrane pressure difference $(\mathrm{Pa})$, and $A$ is the effective membrane area $\left(\mathrm{m}^{2}\right)$.

A mixture of $\mathrm{H}_{2} / \mathrm{CO}_{2}(50 / 50 \mathrm{v} / \mathrm{v})$ was used to evaluate the gas separation performance of the prepared membranes. The prepared membranes were sealed into a home-made stainless-steel permeation cell using the Wicke-Kallenbach technique. ${ }^{33}$ During the measurements, Helium was used as sweep gas in the permeate side and the fluxes were on-line monitored from this side by the gas chromatograph with high precision (GC, Agilent 7820A, Agilent Technologies Inc.). For each sample, the gas permeance and the separation factors of $\mathrm{H}_{2}$ over $\mathrm{CO}_{2}$ were measured at $298 \mathrm{~K}$ and the transmembrane pressure difference was 1.0 bar. The feed flow rate was constant with a total volumetric flow rate of $100 \mathrm{~mL} / \mathrm{min}$ for each gas of $50 \mathrm{~mL} / \mathrm{min}(1 / 1$ mixture). The sweep flow rate was set to $20 \mathrm{~mL} / \mathrm{min}$. The gas permeance was calculated as above and the separation factor $(a)$ for $\mathrm{H}_{2} / \mathrm{CO}_{2}$ mixture was calculated using the following equation (Eq.3), ${ }^{34}$

$\alpha_{\frac{\mathrm{H}_{2}}{\mathrm{CO}_{2}}}=\frac{y_{\mathrm{H}_{2}} / y_{\mathrm{CO}_{2}}}{x_{\mathrm{H}_{2}} / x_{\mathrm{CO}}}(\mathrm{Eq} .3)$

where $y_{\mathrm{H} 2}$ and $y_{\mathrm{CO} 2}$ are the mole fractions of $\mathrm{H}_{2}$ and $\mathrm{CO}_{2}$ in the permeate side, respectively, a $x_{\mathrm{H} 2}$ and $x_{\mathrm{CO} 2}$ are the corresponding mole fractions in the feed side, respectively. The permeation value was averaged from the measurements performed on at least three samples.

DFT Calculations. The energy between HOMO (the highest occupied molecular orbital) and LUMO (the lowest unoccupied molecular orbital) calculations were conducted by $\mathrm{Dmol}^{3}$ in Materials Studio software using the generalized gradient approximation (GGA) based on Perdew-Burke-Ernzerhof (PBE) with spinpolarization. ${ }^{35}$ The double numerical basis set augmented with polarization p-function (DNP) was used to describe the valence orbitals of atoms. And the self-consistent field ( $\mathrm{SCF}$ ) tolerance was set to $10^{-6} \mathrm{Ha}$.

\section{Results and discussion}

Characterization of Bulk CTF-BTD Powder. We chose one triazine-based COF, CTF-BTD (Covalent Triazine Framework-2,1,3-benzothiadiazole-4,7-benzenedicarbonitrile), considering its relatively small pore size and high stability, which are beneficial for practical gas separation. CTFs are a class of COFs based on the cyclotrimerization of nitrile building units, which have been observed to efficiently capture gas, iodine and dye molecules. ${ }^{36}$ CTF-BTD has a hexagonal 2D structure with a pore size of about $0.65 \mathrm{~nm}$ (Figure $1 \mathrm{~A}$ and 
$1 \mathrm{C})$ and a BET surface area of $348 \mathrm{~m}^{2} \mathrm{~g}^{-1}$ (Figure S5 A, Supporting Information). Powder X-ray diffraction (PXRD) patterns are shown in Figure $1 \mathrm{~B}$, as well as the simulated ones with eclipsed stacking structure. A sharp diffraction peak at $6.8^{\circ}$ and a board peak at $26^{\circ}$ correspond to the 100 and 001 crystal planes, respectively. According to d-spacing of 001 crystal plane, the interlayer distance between the adjacent sheets is about $0.34 \mathrm{~nm}$. The SEM images of high magnification of powder demonstrate the 2D layered structure of CTF-BTD (Figure 1 D). Fourier transform infrared spectroscopy (FTIR), $\mathrm{N}_{2}$ adsorption-desorption isotherms, NLDFT pore size distributions and elemental analyses all confirm the successful synthesis of this COF (Figures S5 B,S9 and S11, Supporting Information).

Characterization of CTF-BTD and GO Nanosheets. For successfully preparing 2D nanosheet-based COF membranes by restacking process, it is crucial to obtain high-quality nanosheets with a high lateral size-thickness ratio and flat surface. In this work, we used mechanical wet grinding followed by sonication to disrupt the $\pi$-p noncovalent interactions between layers of CTF-BTD bulk powder. ${ }^{37-38}$ The dispersion solution containing few-layered CTF-BTD nanosheets could be obtained from the supernatant after centrifugation at $8000 \mathrm{rpm}$ to completely separate the large unexfoliated particles. The Tyndall effect is clearly observed in the dispersion solution of CTF-BTD nanosheets (Figure 1 E), indicating the colloidal properties. The transmission electron microscopy (TEM) image shows that the CTF-BTD nanosheets have lateral size of $\sim 2 \mu \mathrm{m}$ (Figure $1 \mathrm{~F}$ ). The crystalline properties of CTF-BTD after exfoliation were confirmed from the high-resolution TEM (HRTEM) image (Figure $1 \mathrm{G}$ ) along with fast inverse Fourier transformation (IFFT) image (Figure $1 \mathrm{H}$ ). A statistical analysis of the AFM images were measured to check the size distribution of the COF nanosheets. The average thickness of these nanosheets is about $2.8 \mathrm{~nm}$ and the average lateral size is about $1.2 \mu \mathrm{m}$, which can be observed by AFM under tapping mode (Figure 1 I and1 J; Figure S6 , Supporting Information). Such high lateral size-thickness ratio ( 500:1) is very helpful for the preparation of ultrathin membranes. To increase the weak force between the COF nanosheets and the inorganic support, the quantitative GO nanosheets were added to CTF-BTD nanosheets dispersion solution during the membrane fabrication process. ${ }^{30}$ Exfoliated GO nanosheets through ultra-sonicating method have average lateral size of $1.0 \mu \mathrm{m}$ and thickness of $1.6 \mathrm{~nm}$ (Figure S7, Supporting Information). The thickness of a single-layer GO nanosheet is $\sim 0.61 \mathrm{~nm}$ in the aqueous phase due to the presence of rich oxygen-containing functional groups and the replacement of $\mathrm{sp}^{3}$ hybridized carbon. ${ }^{39}$ Thus, it reveals that the exfoliated GO nanosheets in this work was about two layers. Meanwhile, we also conducted dynamic light scattering (DLS) to analyze the lateral size of these two nanosheets. The results demonstrate the similar lateral sizes for both CTF-BTD nanosheets $(1.0 \mu \mathrm{m})$ and GO nanosheets $(0.9 \mu \mathrm{m})$ (Figure S8 , Supporting Information).

Characterization of Ultrathin CTF-BTD/GO Membranes. The mixed dispersion liquid of CTFBTD and GO nanosheets was filtered onto the AAO support using a vacuum filtration system to prepare the membranes. To introduce the disturbing process for tuning the restacking of nanosheets, the filtration was performed under different temperatures (293, 333, 373, 413 and $453 \mathrm{~K})$. From the surface SEM images by taking those obtained at $293 \mathrm{~K}$ and $453 \mathrm{~K}$ as examples, it is observed that a dense and continuous layer is successfully formed on the support (Figure 2 A and 2 B). For the membrane prepared at $293 \mathrm{~K}$, the surface is smooth with some slight wrinkles, while the surface of membrane fabricated by $453 \mathrm{~K}$ is a little rough with local unevenness. The cross-sectional SEM images shows that the membranes are well deposited on the support with very regular lamellar stacking of COF nanosheets without defects (Figure 2 C-2 F). The cross-sectional Focused Ion Beam (FIB)-TEM results (Figure $2 \mathrm{G}$ and $\mathbf{2} \mathrm{H}$ ) clearly show the layered morphologies of the CTF-BTD@GO membranes which come from the layered CTF-BTD nanosheets and GO nanosheets. The thicknesses of the prepared ultrathin COF/GO membranes can be tuned from about $140 \mathrm{~nm}$ to $260 \mathrm{~nm}$ through restacking different amounts of COF nanosheets. Statistical analysis of membrane thickness demonstrates that the thickness rarely changes when the membranes were prepared using the same amount of COF nanosheets under different temperatures, revealing that the introduction of thermal perturbation does not influence membrane thickness. The peaks of $1352 \mathrm{~cm}^{-1}$ and $1507 \mathrm{~cm}^{-1}$ in the FT-IR spectra match the characteristic bands of the triazine rings, indicating that the chemical structure of the CTF-BTD nanosheets is preserved during the membrane fabrication process (Figure S12, Supporting Information). The $-\mathrm{OH}$ peak at around $3420 \mathrm{~cm}^{-1}$ in the CTF-BTD/GO membrane has a slight shift (50 
$\mathrm{cm}^{-1}$ ) compared to GO membrane, indicating the interaction between CTF-BTD and GO nanosheets and support through these functional groups. ${ }^{30}$ The XPS spectra of the CTF-BTD/GO membrane showed that the chemical structure of CTF-BTD was not damaged after the membrane fabrication process (Figure S10 , Supporting Information).

PXRD and Raman spectroscory were measured to characterize the difference in the membrane structures induced by thermal perturbation. FromFigure $4 \mathrm{~A}$, it can be seen that there is a small shift $\left(0.2^{\circ}\right)$ of $2 \vartheta$ to higher angle in the PXRD pattern, from $6.8^{\circ}$ for CTF-BTD/GO membrane prepared at $293 \mathrm{~K}$ to $7.0^{\circ}$ for that prepared at $453 \mathrm{~K}$. This phenomenon demonstrated that the interplanar distances along the 100 direction in CTF-BTD/GO-453K membrane are smaller than those in CTF-BTD/GO-293K one. In fact, similar shift in PXRD was observed in the twisted and untwisted restacking of 2D MOF nanosheets in our recent work. ${ }^{40}$ In Raman spectroscopy (Figure $4 \mathrm{~B}$ ), the value of $\mathrm{I}_{\mathrm{D}} / \mathrm{I}_{\mathrm{G}}$ is increased from 1.61 to 1.72 with the change of preparation temperature (from $293 \mathrm{~K}$ to $453 \mathrm{~K}$ ), suggesting a less organized carbon structure. ${ }^{41}$ In consideration of the same thickness of membrane, the differences in PXRD and Raman spectra may be due to the change of the relative position of restacked nanosheets in the horizontal direction along the surface of support, caused by thermal perturbation using different temperatures.

Gas Separation Performance. To further determine the different microstructures of the obtained ultrathin COF membranes, single-component gas permeation and mixed gas separationwere respectively measured by soap-bubble flow apparatus and custom-built Wicke-Kallenbach setup. The transmembrane pressure difference between the feed side and permeate side was kept to be 1.0 bar. Before measurement, the membrane was activated at $313 \mathrm{~K}$ under vacuum for $24 \mathrm{~h}$. To guarantee the reproducibility of the performance, at least three tests were conducted and the results of error range are shown in theFigure $\mathbf{3}$ and Table S2 (Supporting Information). At the first step, we studied the effect of both CTF-BTD nanosheets (CONs) amount and GO amount on the permeation performance on the condition that the GO amount is fixed at $0.008 \mathrm{mg}$ (Figure $3 \mathrm{~A}$ and $\mathbf{3} \mathrm{B}$ ), as optimized in our previous work. ${ }^{30}$ It can be seen that with the increase of CTF-BTD nanosheet amount from $0.05 \mathrm{mg}$ to $0.2 \mathrm{mg}, \mathrm{H}_{2}$ permeance decreases from 2347.0 GPU to 923.2 GPU and the corresponding $\mathrm{H}_{2} / \mathrm{CO}_{2}$ selectivity increases from 12.6 to 23.4. Further increasing the amount of CTF-BTD nanosheets may bring difficulties to form dense and continuous restacking layer on the support, inducing the $\mathrm{H}_{2}$ permeance decreases significantly with the corresponding decrease of selectivity. Similarly, the $\mathrm{H}_{2}$ permeance decreases and $\mathrm{H}_{2} / \mathrm{CO}_{2}$ selectivity increases from 16.3 to 23.4 with increasing the GO amount from $0.006 \mathrm{mg}$ to $0.008 \mathrm{mg}$. Further increasing the amount of GO amount brings a sharp decline of permeance for both $\mathrm{H}_{2}$ and $\mathrm{CO}_{2}$. This is because the more nonporous GO sheets may block more gas transport pathways. Although both gas permeances decrease, different decreasing rates for these two gases are observed where GO amount is less or more than $0.008 \mathrm{mg}$, resulting in a maximum $\mathrm{H}_{2} / \mathrm{CO}_{2}$ ideal selectivity when GO amount is $0.008 \mathrm{mg}$.

Effect of Thermal Perturbation for CTF-BTD/GO Membrane. In order to study the effect of thermal perturbation on the restacking of nanosheets and the resulted performance, we prepared membranes under different filtration temperatures using the same amount of building nanosheets in $250 \mathrm{~mL}$ of mixed solvent on the basis of the previously optimized amounts (CTF-BTD: $0.2 \mathrm{mg}$, GO: $0.008 \mathrm{mg}$ ). As shown inFigure $3 \mathrm{C}$, with the filtration temperature increasing from $293 \mathrm{~K}$ to $453 \mathrm{~K}$, both $\mathrm{H}_{2}$ and $\mathrm{CO}_{2}$ permeances decrease while an increasing $\mathrm{H}_{2} / \mathrm{CO}_{2}$ ideal selectivity is observed, which is resulted from the more decrease of $\mathrm{CO}_{2}$ permeance. The excellent separation performance of CTF-BTD/GO-0.2-0.008-453 membrane surpass not only the 2008 Robeson upper bound of conventional polymer membranes for $\mathrm{H}_{2} / \mathrm{CO}_{2}$ (assuming the thickness is $200 \mathrm{~nm}),{ }^{42}$ but also the performance of a wide range of reported membranes, such as polymers, MOFs, COFs and their hybrid membranes (Figure $3 \mathrm{~F}$ ). ${ }^{8,20,30,43-48}$ Considering the probable chemical change of GO nanosheets with abundant surface functional groups during the membrane fabrication at a relatively high temperature, a set of control experiments were carried out by pretreating GO nanosheets at $453 \mathrm{~K}$, followed by the filtration process with CTF-BTD nanosheets as mentioned above. It is observed that the separation performance does not change much with respect to the membrane prepared without the pretreating of GO (Table S3, Supporting Information). Therefore, the large decrement in $\mathrm{CO}_{2}$ permeance is due to the restacking of nanosheets induced by heating filtration. PXRD and Raman results have indicated 
that the introduction of thermal perturbation directly affects the microstructure between the nanosheets in the restacking process. Since these two membranes prepared at different temperatures are with the similar thickness $(260 \mathrm{~nm})$, we preferentially deduce that different permeation performance is a result of different restacking modes in the horizontal direction along the surface of support. By vacuum filtration procedure, the ultrathin COF nanosheets are not only subjected to the pulling force in the vertical direction, but also obtained the energy from the heating movement turbulently in the horizontal direction, resulting in re-arrangement of the COF nanosheets in a more random and irregular way. Besides, the faster evaporation of solvent under high temperature is also beneficial to the disorder restacking of the nanosheets. Therefore, more distorted narrow molecular transport channels can be created in adjacent layers by strenuous motion. As a result, an enhanced $\mathrm{H}_{2} / \mathrm{CO}_{2}$ ideal selectivity (43.2) with high $\mathrm{H}_{2}$ permeance (655.6 GPU) is observed, in comparison to the membrane prepared under room temperature with a $\mathrm{H}_{2} / \mathrm{CO}_{2}$ ideal selectivity of 23.4 and $\mathrm{H}_{2}$ permeance of $845.8 \mathrm{GPU}$. When the filtration temperature is further increased to $483 \mathrm{~K}$, the solvent evaporation is more vigorously, so that the membrane may have several defects, which inversely induces the increased permeance and reduced selectivity. Meanwhile, gas permeances and $\mathrm{H}_{2} / \mathrm{CO}_{2}$ ideal selectivity of CTF-BTD/GO membranes with CTF-BTD nanosheets amount of $0.05 \mathrm{mg}, 0.10 \mathrm{mg}, 0.15 \mathrm{mg}$ and $0.25 \mathrm{mg}$ were also studied, as shown inFigure S13 (Supporting Information), exhibiting the same trend of separation performance upon the thermal perturbation. Similarly, enhanced ideal selectivity for $\mathrm{H}_{2} / \mathrm{N}_{2}$ and $\mathrm{H}_{2} / \mathrm{CH}_{4}$ gas pairs are also observed, displaying the increase from 12.7 to 18.0 and 17.9 to 26.1, respectively (Figure $3 \mathrm{D}$ and $3 \mathrm{E}$ ). These results further demonstrated the effectiveness adjustment of thermal perturbation on the microstructures of the obtained ultrathin COF membranes, which indicates the realization of horizontal restacking control suggested by our theoretical design. ${ }^{29}$ Thus, ultrathin $2 \mathrm{D}$ COF membranes can form an energetic microenvironment around the narrow interlayer passages through tuning restacking mode along vertical or horizontal direction to tuning separation performance.

To further investigate the different position of nanosheets in the horizontal direction along the surface of support resulted by the restacking process, the composite membranes were characterized by the solid fluorescence analysis, considering the organic units in CTF-BTD. As shown in Figure 4 C, there is obvious difference of fluorescence between CTF-BTD/GO-0.2-0.008-293 membrane and CTF-BTD/GO-0.2-0.008-453 membrane upon excitation wavelength at $280 \mathrm{~nm}$, and excitation wavelength was measured by UV-visible spectrophotometer. The membrane prepared at $453 \mathrm{~K}$ exhibits a strong fluorescence intensity compared to that prepared at $293 \mathrm{~K}$. To explain this phenomenon, the HOMO and LUMO orbital energies were calculated by density functional theory method in a simple model with two CTF-BTD single layers. As shown in Figure 4D andTable S4 (Supporting Information), the LUMO-HOMO energy gap is gradually decreased with the increase of the change of the relative displacement of these two COF sheets in the horizontal direction, because of the varied out-plane $\pi-\pi$ interaction between the aromatic rings on the adjacent COF sheets. Small LUMO-HOMO energy gap is favorable for fluorescence emission. ${ }^{49}$ In the work of Albacete et al., ${ }^{50}$ it is observed that IMDEA-COF- 1 can minimize the $\pi-\pi$ interaction between the pyrene units to turn on the fluorescence due to its AB-stacked structure compared to AA-stacked counterpart IMDEA-COF-2. This gives an indirect evidence for the change in the relative position between nanosheets formed under thermal perturbation, thereby realizing the membrane separation performance tunable in the horizontal orientation (Figure 4E ).

Furthermore, the separation of mixed gas $\mathrm{H}_{2} / \mathrm{CO}_{2}(50 / 50, \mathrm{v} / \mathrm{v})$ was conducted to evaluate the practical potential of the membranes since the separation of this mixture is one of the important pre-combustion $\mathrm{CO}_{2}$ capture processes. As shown in Figure S14(Supporting Information), $\mathrm{H}_{2}$ permeance and $\mathrm{H}_{2} / \mathrm{CO}_{2}$ separation factor of CTF-BTD/GO-0.2-0.008-453 membrane is 577.3 GPU and 36.8, respectively. These are close to the results obtained in single gas permeation measurement with a slight decrease.

\section{Conclusions}

In summary, we realized horizontal restacking control of CONs and GO nanosheet composite membranes with the aid of introducing thermal perturbation through a heating vacuum filtration process. The interlayer microstructure of the resulted CTF-BTD/GO membranes became complex interlacing. As a result, the gas 
selectivities of $\mathrm{H}_{2}$ over other gases were enhanced from 23.4 to $43.2,12.7$ to 18.0, and 17.9 to 26.1 for $\mathrm{H}_{2} / \mathrm{CO}_{2}$, $\mathrm{H}_{2} / \mathrm{N}_{2}$, and $\mathrm{H}_{2} / \mathrm{CH}_{4}$ gas pairs, exhibiting competitive advantages over other membranes. Meanwhile, thanks to the ultrathin properties, the $\mathrm{H}_{2}$ permeance of CTF-BTD/GO-0.2-0.008-453 membrane can reach as high as 655.6 GPU. These results not only recommend the CTF-BTD/GO membranes as promising candidates for $\mathrm{H}_{2}$ purification, syngas separation, nature gas purification, but also demonstrate the heating vacuum filtration is an effective strategy to optimize the restacking microstructure of other $2 \mathrm{D}$ material related membranes.

\section{Associated content}

Supporting Information : Schematic diagram of the experimental setup for single-gas permeation tests, Synthetic Procedure of monomer BTD, adsorption isotherms bulk CTF-BTD powder, AFM image of CTFBTD and GO nanosheets, DLS size distribution of CTF-BTD and GO, XPS spectra of the CTF-BTD powder and fabricated CTF-BTD/GO membranes, XPS spectra of the fabricated CTF-BTD/GO membranes and CTF-BTD powder, FT-IR spectra of CTF-BTD/GO membranes, gas permeance and $\mathrm{H}_{2} / \mathrm{CO}_{2}$ selectivity of CTF-BTD/GO membranes with different CTF-BTD nanosheets loading amount, membranes gas separation performance prepared by pretreatment and without pretreatment of $\mathrm{GO}$, gas permeance and $\mathrm{H}_{2} / \mathrm{CO}_{2}$ separation factor of CTF-BTD/GO membranes using mixed gases, simulation calculation about HOMO and LUMO orbital energies of CTF-BTD materials, summary of COF based membranes and other ultrathin membranes for gas separation performance reported in recent years, study on the universality of the heating filtration process method.

\section{Author information}

Corresponding Author

*Corresponding author. E-mail: liudh@mail.buct.edu.cn

Author Contributions

\#These authors contributed equally. D.L. designed the work and wrote the paper; Y.Z. and P.L. conducted the experiments; Y.Y. analyzed the data and wrote the paper; Y.Z. and K.W. conducted the calculations; D.L. and D.Z. discussed the results.

Notes

The authors declare no competing financial interest.

\section{Acknowledgment}

The financial support of the National Natural Science Foundation of China (Nos. 21722602 and 21978005) is greatly appreciated.

\section{References}

1. Sholl DS, Lively RP. Seven chemical separations to change the world. Nature2016;532:435-437.

2. Kang Z, Fan L, Sun D. Recent advances and challenges of metal-organic framework membranes for gas separation. J. Mater. Chem. A2017;5:10073-10091.

3. Lin JY. Molecular sieves for gas separation. Science 2016;353:121-122.

4. Wang S, Yang L, He G, Shi B, Li Y, Wu H, Zhang R, Nunes S, Jiang Z. Two-dimensional nanochannel membranes for molecular and ionic separations. Chem. Soc. Rev. 2020;49:1071-1089.

5. Yousefi N, Lu X, Elimelech M, Tufenkji N. Environmental performance of graphene-based 3D macrostructures. Nat. Nanotechnol.2019;14:107-119.

6. Shinde D, Sheng G, Li X, Ostwal M, Emwas AH, Huang K, Lai Z. Crystalline 2D covalent organic framework membranes for high-flux organic solvent nanofiltration. J. Am. Chem. Soc.2018;140:1434214349.

7. Yao J, Wang H. Zeolitic imidazolate framework composite membranes and thin films: synthesis and applications. Chem. Soc. Rev.2014;43:4470-4493. 
8. Li H, Song Z, Zhang X, Huang Y, Li S, Mao Y, Ploehn HJ, Bao Y, Yu M. Ultrathin molecular-sieving graphene oxide membranes for selective hydrogen separation. Science 2013;342:95-98.

9. Kang K, Lee KH, Han Y, Gao H, Xie S, Muller DA, Park J. Layer-by-Layer Assembly of twodimensional materials into wafer-scale heterostructures. Nature 2017;550:229-233.

10. Yang Y, Yang X, Liang L, Gao Y, Cheng H, Li X, Zou M, Ma R, Yuan Q, Duan X. Large-area graphene-nanomesh/carbon-nanotube hybrid membranes for ionic and molecular nanofiltration. Science 2019;364:1057-1062.

11. Min B, Yang S, Korde A, Kwon YH, Jones CW, Nair S. Continuous zeolite MFI membranes fabricated from 2D MFI nanosheets on ceramic hollow fibers. Angew. Chem. Int. Ed. 2019;58:8201-8205.

12. Yang X, Yang Y, Fu L, Zou M, Li Z, Cao A, Yuan Q. An ultrathin flexible 2D membrane based on single-walled nanotube- $\mathrm{MoS}_{2}$ hybrid film for high-performance solar steam generation. Adv. Funct. Mater. 2018;28:1704505.

13. Dou H, Jiang B, Xu M, Zhang Z, Wen G, Peng F, Yu A, Bai Z, Sun Y, Zhang L, Jiang Z, Chen Z. Boron nitride membranes with a distinct nanoconfinement effect for efficient ethylene/ethane separation.Angew. Chem. Int. Ed. 2019;131:14107-14113.

14. Wang N, Huang Z, Li X, Li J, Ji S, An Q. Tuning molecular sieving channels of layered double hydroxides membrane with direct intercalation of amino acids. J. Mater. Chem. A2018;6:1714817155 .

15. Liang B, Wang H, Shi X, Shen B, He X, Ghazi ZA, Khan NA, Sin H, Khattak A, Li L. Tang Z.. Microporous membranes comprising conjugated polymers with rigid backbones enable ultrafast organicsolvent nanofiltration. Nat. Chem. 2018;10:961-967.

16. Xu G, Yamada T, Otsubo K, Sakaida S, Kitagawa H. Facile "modular assembly" for fast construction of a highly oriented crystalline MOF nanofilm. J. Am. Chem. Soc. 2012;134:16524-16527.

17. Hmadeh M, Lu Z, Liu Z, Gandara F, Furukawa H, Wan S, Angustyn V, Chang R, Liao L, Zhou F, Perre E, Ozolins V, Suenaga K, Duan X, Dunn B, Yamamto Y, Terasaki O, Yaghi O. M. New porous crystals of extended metal-catecholates. Chem. Mater. 2012;24:3511-3513.

18. Yao, M, Xiu J, Huang Q, Li W, Wu W, Wu A, Cao L, Deng W, Wang G, Xu G. Van der waals heterostructured MOF-on-MOF thin films:cascading functionality to realize advanced chemiresistive sensing. Angew. Chem. Int. Ed. 2019;58:14915-14919.

19. Wu J, Chen J, Wang C, Zhou Y, Ba K, Xu H, Bao W, Xu X, Carlsson A, Lazar S, Meingast A, Sun Z, Deng H. Metal-organic framework for transparent electronics. Adv. Sci. 2020;7:1903003.

20. Shan M, Liu X, Wang X, Yarulina I, Seoane B, Kapteijn F, Gascon J. Facile manufacture of porous organic framework membranes for precombustion $\mathrm{CO}_{2}$ capture. Sci. Adv.2018;4:eaau1698.

21. Franco C, Rodríguez-San-Miguel D, Sorrenti A, Sevim S, Pons R, Platero-Prats AE, Pavlovic M, Szilágyi I, Ruiz Gonzalez ML, González-Calbet JM, Bochicchio D, Pesce, L, Pavan GM, Imaz I, CanoSarabia M, Maspoch D, Pané S, de Mello AJ, Zamora F, Puigmartí-Luis J. Biomimetic synthesis of sub-20 nm covalent organic frameworks in water. J. Am. Chem. Soc. 2020;142:3540-3547.

22. Yang H, Yang L, Wang H, Xu Z, Zhao Y, Luo Y, Nasir N, Song Y, Wu H, Pan F, Zhong Y. Covalent organic framework membranes through a mixed-dimensional assembly for molecular separations. Nat. Commun. 2019;10:1-10.

23. Ying Y, Tong M, Ning S, Ravi SK, Peh SB, Tan S, Pennycook SJ, Zhao D. Ultrathin two-dimensional membranes assembled by ionic covalent organic nanosheets with reduced apertures for gas separation. J. Am. Chem. Soc. 2020;142:4472-4480.

24. Zhang $\mathrm{C}$, Wu B, Ma M, Wang Z, Xu Z. Ultrathin metal/covalent-organic framework membranes towards ultimate separation. Chem. Soc. Rev. 2019;48:3811-3841.

25. Huang N, Wang P, Jiang D. Covalent organic frameworks: a materials platform for structural and functional designs. Nat. Rev. Mater. 2016;10:1-19.

26. Geng K, He T, Liu R, Dalapati S, Tan K, Li Z, Tao S, Gong Y, Jiang Q, Jiang D. Covalent organic frameworks: design, synthesis, and functions. Chem. Rev. 2020;120:8814-8933.

27. Li G, Zhang K, Tsuru T. Two-dimensional covalent organic framework (COF) membranes fabricated via the assembly of exfoliated COF nanosheets. ACS Appl. Mater. Interf. 2017;9:8433-8436. 
28. Lohse MS, Bein T. Covalent organic frameworks: structures, synthesis, and applications. Adv. Funct. Mater. 2018;28:1705553.

29. Tong M, Yang Q, Ma Q, Liu D, Zhong C. Few-layered ultrathin covalent organic framework membranes for gas separation: a computational study.J. Mater. Chem. A 2016;4:124-131.

30. Ying Y, Liu D, Ma J, Tong M, Zhang W, Huang H, Yang Q, Zhong C. A GO-assisted method for the preparation of ultrathin covalent organic framework membranes for gas separation. J. Mater. Chem. A2016;4:13444-13449.

31. Agneeswari R, Tamilavan V, Song M, Hyun MH. Property modulation of benzodithiophene-based polymers via the incorporation of a covalently bonded novel 2, 1, 3-benzothiadiazole-1, 2, 4-oxadiazole derivative in their main chain for polymer solar cells. J. Mater. Chem. C2014;2:8515-8524.

32. Huang Y, Liu D, Liu Z, Zhong C. Synthesis of Zeolitic imidazolate framework membrane using temperature-switching synthesis strategy for gas separation. Ind. Eng. Chem. Res. 2016;55:7164-7170.

33. Ban Y, Li Y, Peng Y, Jin H, Jiao W, Liu X, Yang W. Metal-substituted zeolitic imidazolate framework ZIF-108: gas-sorption and membrane-separation properties. Chem.-Eur. J.2014;20:11402-11409.

34. Guo X, Huang H, Ban Y, Yang Q, Xiao Y, Li Y, Yang W, Zhong C. Mixed matrix membranes incorporated with amine-functionalized titanium-based metal-organic framework for $\mathrm{CO}_{2} / \mathrm{CH}_{4}$ separation. J. Membr. Sci. 2015;478:130-139.

35. Perdew, J. P.; Burke, K.; Ernzerhof, M. Generalized gradient approximation made simple. Phys. Rev. Lett. 1996;77:3865.

36. Kuhn P, Antonietti MA, Thomas A. Porous, covalent triazine-based frameworks prepared by ionothermal synthesis. Angew. Chem. Int. Ed. 2008;47:3450-3453.

37. Bunck DN, Dichtel WR. Bulk synthesis of exfoliated two-dimensional polymers using hydrazone-linked covalent organic frameworks. J. Am. Chem. Soc. 2013;135:14952-14955.

38. Berlanga I, Ruiz-Gonzalez ML, Gonzalez-Calbet JM, Fierro JLG, Mas-Balleste R, Zamora F. Delamination of layered covalent organic frameworks. Small 2011; 7: 1207-1211.

39. Stankovich S, Dikin DA, Piner RD, Kohlhaas KA, Kleinhammes A, JiaY, Wu Y, Nguyen ST, Ruoff RS. Synthesis of graphene-based nanosheets via chemical reduction of exfoliated graphite oxide. Carbon 2007;45:1558-1565.

40. Tao Z, Wu J, Zhao Y, Xu M, Tang W, Zhang Q, Gu L, Liu D, Gu Z. Untwisted restacking of twodimensional metal-organic framework nanosheets for highly selective isomer separations. Nat. Commun. 2019;10:1-8.

41. Briceno K, Montane D, Garcia-Valls R, Iulianelli A, Basile A. Fabrication variables affecting the structure and properties of supported carbon molecular sieve membranes for hydrogen separation.J. Membr. Sci. 2012;415:288-297.

42. Robeson LM. The upper bound revisited. J. Membr. Sci.2008;320:390-400.

43. Peng Y, Li, Y, Ban Y, Jin H, Jiao W, Liu X, Yang W. Metal-organic framework nanosheets as building blocks for molecular sieving membranes. Science 2014;346:1356-1359.

44. Achari A, Sahana S, Eswaramoorthy M. High performance $\mathrm{MoS}_{2}$ membranes: effects of thermally driven phase transition on $\mathrm{CO}_{2}$ separation efficiency. Energ. Environ. Sci. 2016;9:1224-1228.

45. Fan H, Mundstock A, Feldhoff A, Knebel A, Gu J, Meng H, Caro JR. Covalent organic frameworkcovalent organic framework bilayer membranes for highly selective gas separation. J. Am. Chem. Soc. 2018;140:10094-10098.

46. Fu J, Das S, Xing G, Ben T, Valtchev V, Qiu S. Fabrication of COF-MOF composite membranes and their highly selective separation of $\mathrm{H}_{2} / \mathrm{CO}_{2}$. J. Am. Chem. Soc.2016;138:7673-7680.

47. Shen J, Liu G, Huang K, Chu Z, Jin W, Xu N. Subnanometer two-dimensional graphene oxide channels for ultrafast gas sieving. ACS Nano 2016;10:3398-3409.

48. Wang X, Chi L, Zhang K, Qian Y, Gupta KM, Kang Z, Jiang J, Zhao D. Reversed thermo-switchable molecular sieving membranes composed of two-dimensional metal-organic nanosheets for gas separation.Nat. Commun. 2017;8:1-10.

49. Nagarkar SS, Joarder B, Chaudhari AK, Mukherjee S, Ghosh SK. Highly selective detection of nitro explosives by a luminescent metal-organic framework. Angew. Chem. Int. Ed. 2013;52:2881-2885. 
50. Albacete P, Martinez JI, Li X, Lopez-Moreno A, Mena-Hernando S, Platero-Prats AE, Montoro C, Loh KP, Perez EM, Zamora F. Layer-stacking-driven fluorescence in a two-dimensional imine-linked covalent organic framework. J. Am. Chem. Soc.2018;140:12922-12929.

\section{List of Figure Captions}

Scheme 1. Illustration of the fabrication of ultrathin COF membranes with controling horizontal restacking by introducing thermal perturbation.

Figure 1. Characterization of the bulk powder and exfoliated CTF-BTD nanosheets. (A) The synthesis route of the CTF-BTD by a cyclotrimerization reaction. (B) PXRD patterns of bulk CTF-BTD powder. (C) Eclipsed stack of 2D sheets and microporous channels in CTF-BTD. Colors used for identification: C gray, $\mathrm{N}$ blue, $\mathrm{S}$ yellow, $\mathrm{H}$ white. (D) SEM image of bulk CTF-BTD powder. The insert is the high-magnification SEM image of the selected area. The CTF-BTD nanosheets (CONs) for (E) Tyndall effect of the dispersion solution; (F) TEM image; (G) HRTEM image and (H) IFFT image and the inset in picture (H) is the corresponding FFT image; (I) AFM image and (J) the corresponding height profiles.

Figure 2. SEM and TEM images of CTF-BTD/GO membranes. Top surface SEM image: (A) CTFBTD/GO-0.1-0.004-293, and (B) CTF-BTD/GO-0.1-0.004-453. Cross sectional SEM iamge: (C) CTFBTD/GO-0.1-0.004-293, (D) CTF-BTD/GO-0.1-0.004-453, (E) CTF-BTD/GO-0.2-0.008-293, and (F) CTF$\mathrm{BTD} / \mathrm{GO}-0.2-0.008-453$. Inset: digital images of the prepared membranes on AAO support (25 $\mathrm{mm}$ in diameter). Cross sectional TEM iamge: (G) CTF-BTD/GO-0.2-0.008-293, and (H) CTF-BTD/GO-0.2$0.008-453$.

Figure 3. Gas permeance and $\mathrm{H}_{2} / \mathrm{CO}_{2}$ ideal selectivity of CTF-BTD/GO membranes. (A) The effect of CTF-BTD nanosheets amount when keeping GO amount as $0.008 \mathrm{mg}$. (B) The effect of GO nanosheets amount when keeping CTF-BTD amount as $0.2 \mathrm{mg}$. (C) The effect of preparation temperature when keeping CTF-BTD and GO nanosheets amount as $0.2 \mathrm{mg}$ and $0.008 \mathrm{mg}$. (D) Single-component gas $\left(\mathrm{H}_{2}\right.$, $\mathrm{CO}_{2}, \mathrm{~N}_{2}, \mathrm{CH}_{4}$ ) permeances. (E) The ideal selectivities for $\mathrm{H}_{2}$ over other gases. (F) Comparison of CTFBTD/GO ultrathin membranes performance $\left(\mathrm{H}_{2} / \mathrm{CO}_{2}\right.$ ideal selectivity vs $\mathrm{H}_{2}$ permeance) with other reported membranes. The upper bound for $\mathrm{H}_{2} / \mathrm{CO}_{2}$ is based on the Robeson upper bound (2008) for polymeric membrane assuming the thickness is $200 \mathrm{~nm}$.

Figure 4. Characterizations and Calculations. Characterization of the ultrathin CTF-BTD/GO membranes: (A) PXRD patterns; (B) Raman spectra and (C) the fluorescence emission spectra upon excitation wavelength at $280 \mathrm{~nm}$ (left one is the excitation spectrum). (D) The HOMO and LUMO orbital energies of different stacking structures for CTF-BTD nanosheets. (E) Illustration of the variation of the interlayer passage in the membrane with orderly and disorderly overlapped layers. 


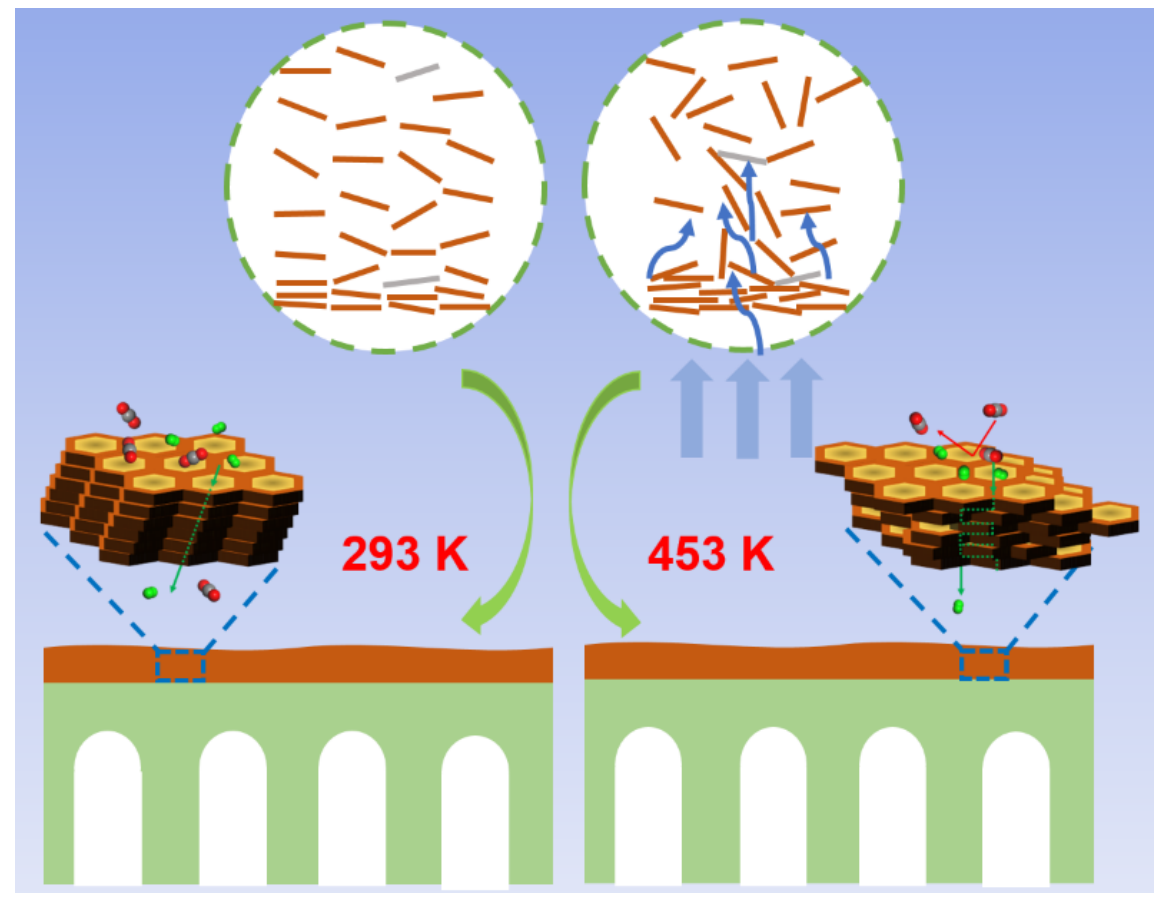

Scheme 1. Illustration of the fabrication of ultrathin COF membranes with controling horizontal restacking by introducing thermal perturbation. 

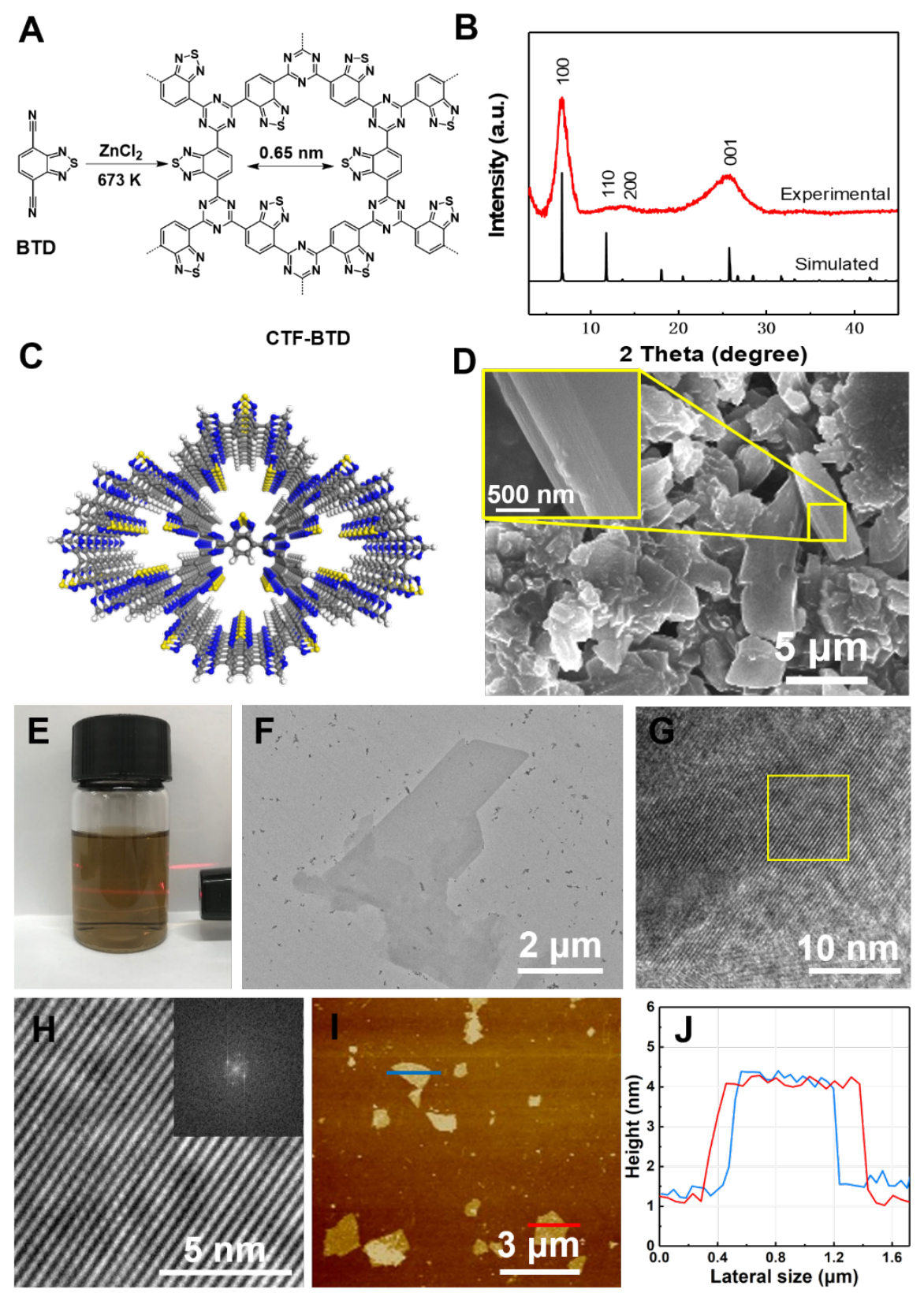

Figure 1. Characterization of the bulk powder and exfoliated CTF-BTD nanosheets. (A) The synthesis route of the CTF-BTD by a cyclotrimerization reaction. (B) PXRD patterns of bulk CTF-BTD powder. (C) Eclipsed stack of 2D sheets and microporous channels in CTF-BTD. Colors used for identification: C gray, $\mathrm{N}$ blue, $\mathrm{S}$ yellow, $\mathrm{H}$ white. (D) SEM image of bulk CTF-BTD powder. The insert is the high-magnification SEM image of the selected area. The CTF-BTD nanosheets (CONs) for (E) Tyndall effect of the dispersion solution; (F) TEM image; (G) HRTEM image and (H) IFFT image and the inset in picture (H) is the corresponding FFT image; (I) AFM image and (J) the corresponding height profiles. 


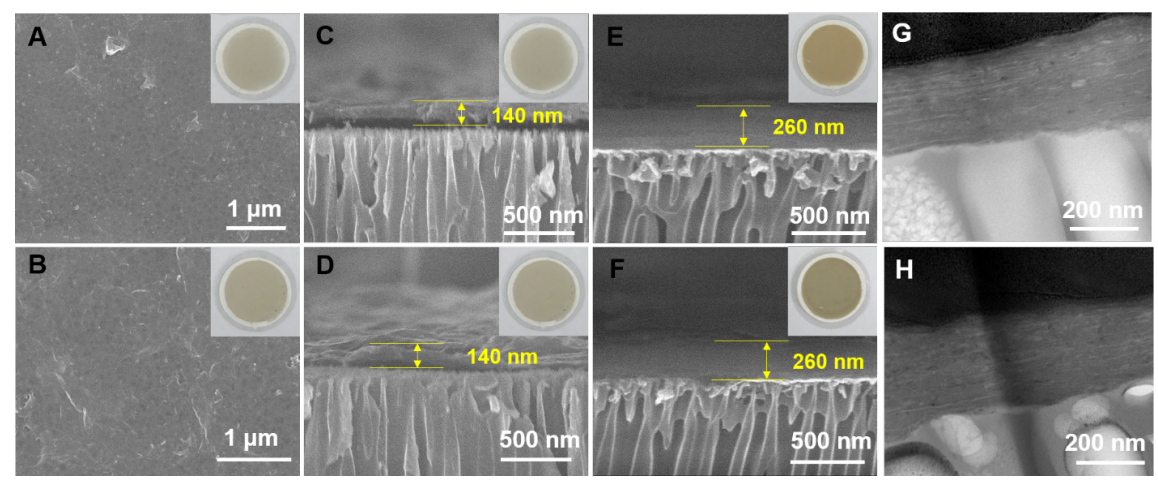

Figure 2. SEM and TEM images of CTF-BTD/GO membranes. Top surface SEM image: (A) CTFBTD/GO-0.1-0.004-293, and (B) CTF-BTD/GO-0.1-0.004-453. Cross sectional SEM iamge: (C) CTFBTD/GO-0.1-0.004-293, (D) CTF-BTD/GO-0.1-0.004-453, (E) CTF-BTD/GO-0.2-0.008-293, and (F) CTF$\mathrm{BTD} / \mathrm{GO}-0.2-0.008-453$. Inset: digital images of the prepared membranes on AAO support (25 $\mathrm{mm}$ in diameter). Cross sectional TEM iamge: (G) CTF-BTD/GO-0.2-0.008-293, and (H) CTF-BTD/GO-0.20.008-453.
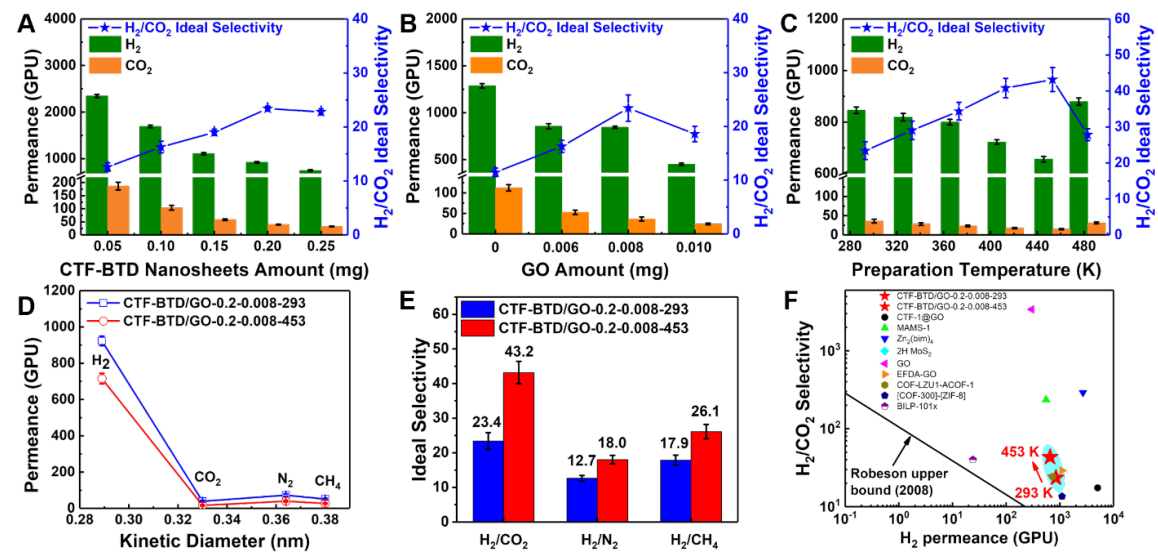

Figure 3. Gas permeance and $\mathrm{H}_{2} / \mathrm{CO}_{2}$ ideal selectivity of CTF-BTD/GO membranes. (A) The effect of CTF-BTD nanosheets amount when keeping GO amount as $0.008 \mathrm{mg}$. (B) The effect of GO nanosheets amount when keeping CTF-BTD amount as $0.2 \mathrm{mg}$. (C) The effect of preparation temperature when keeping CTF-BTD and GO nanosheets amount as $0.2 \mathrm{mg}$ and $0.008 \mathrm{mg}$. (D) Single-component gas $\left(\mathrm{H}_{2}\right.$, $\mathrm{CO}_{2}, \mathrm{~N}_{2}, \mathrm{CH}_{4}$ ) permeances. (E) The ideal selectivities for $\mathrm{H}_{2}$ over other gases. (F) Comparison of CTFBTD/GO ultrathin membranes performance $\left(\mathrm{H}_{2} / \mathrm{CO}_{2}\right.$ ideal selectivity vs $\mathrm{H}_{2}$ permeance) with other reported membranes. The upper bound for $\mathrm{H}_{2} / \mathrm{CO}_{2}$ is based on the Robeson upper bound (2008) for polymeric membrane assuming the thickness is $200 \mathrm{~nm}$. 

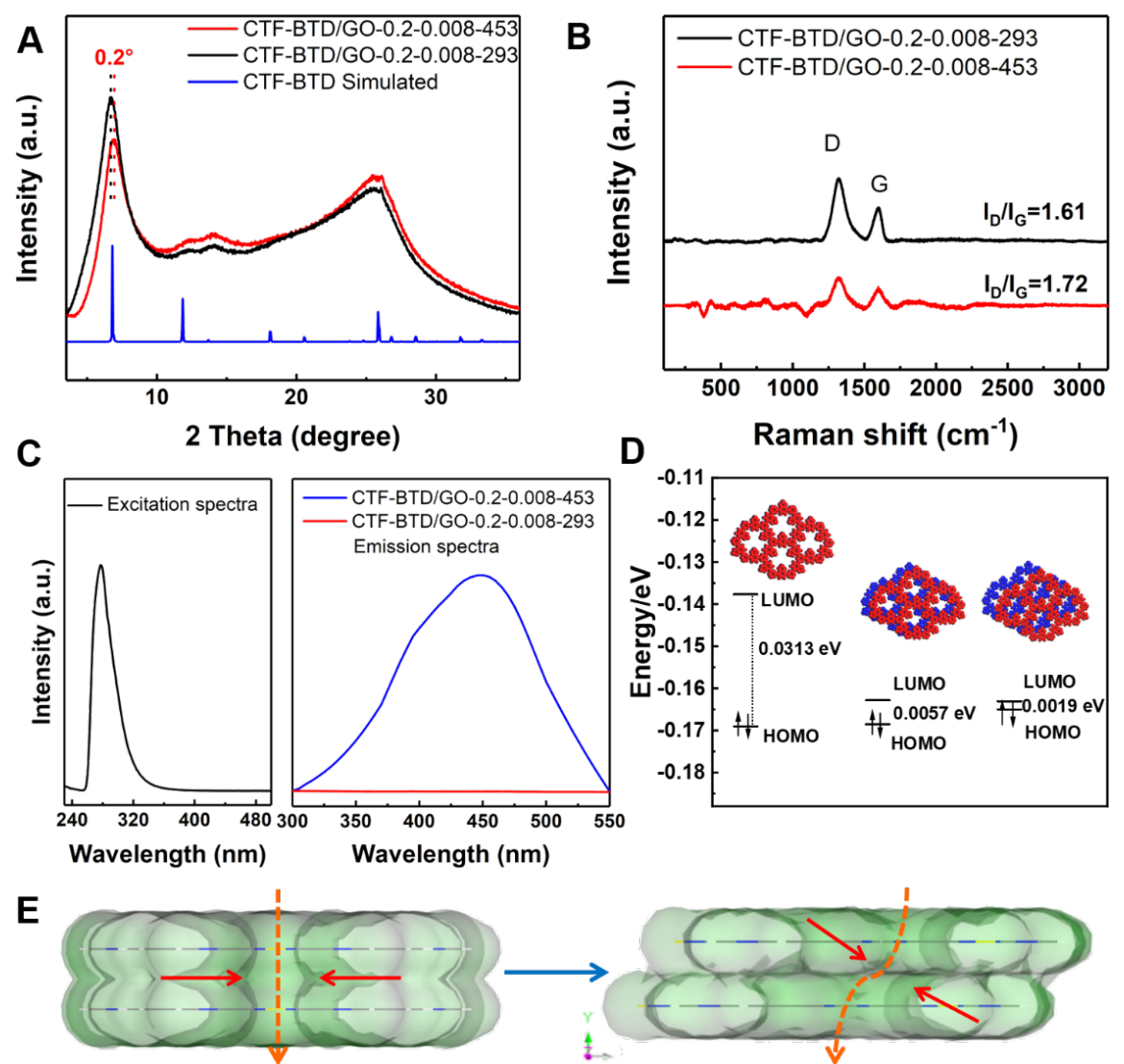

Interlayer passage

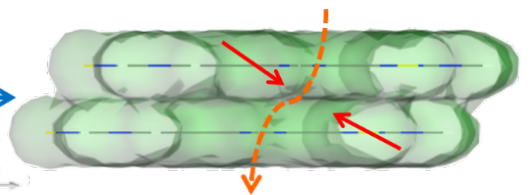

Narrow interlayer passage

Figure 4. Characterizations and Calculations. Characterization of the ultrathin CTF-BTD/GO membranes: (A) PXRD patterns; (B) Raman spectra and (C) the fluorescence emission spectra upon excitation wavelength at $280 \mathrm{~nm}$ (left one is the excitation spectrum). (D) The HOMO and LUMO orbital energies of different stacking structures for CTF-BTD nanosheets. (E) Illustration of the variation of the interlayer passage in the membrane with orderly and disorderly overlapped layers. 